shorter average actual sleeping time than no visual fatigue $(\mathrm{p}<$ 0.05 ), and the visual fatigue could be a predictor to average actual sleeping time.

Conclusions Health surveillance for VDU workers should include not only visual fatigue, but also extra-ocular symptoms, especially in upper limb disorders, and sleep problems. Designing better work-rest schedule and ergonomic workplace may be the preventive strategies at this workplace. Future research with a longitudinal study design is needed to study the psychosocial stress, productivity effects after visual fatigue on VDU workers.

\section{ASSOCIATION BETWEEN LIFESTYLE BEHAVIORS AND BODY WEIGHT CHANGES IN MIDDLE-AGED TAIWANESE MALE WORKERS: A 5-YEAR OBSERVATIONAL COHORT STUDY}

1) H Lee, ${ }^{1}$ Chang, ${ }^{2}$ Wang, ${ }^{3}$ Hsiao. ${ }^{1}$ National Health Research Institutes, Zhuna, Taiwan; ${ }^{2}$ Department of Public Health, College of Medicine, National Cheng Kung University, Tainan, Taiwan; ${ }^{3}$ Hsiao's Charity Clinic, Taoyuan, Taiwan

\subsection{6/oemed-2013-101717.154}

Objectives To investigate association between lifestyle factors and 5 -year body weight gain among apparently healthy middleaged male workers.

Methods A occupational cohort in an electronic-manufacturing factory was established by using a medical checkup program in 2002, and was followed up with a health examination in 2007. Baseline comprehensive questionnaires were applied for collection of lifestyle behaviours, and abdominal ultrasound was performed for evaluation of hepatic steatosis. Male worker with complete data of baseline lifestyle factors, and laboratory examination, including ALT, ferritin, were included in analysis. Crosssectional analyses in 2002 were conducted by stepwise multiple linear regressions for exploring significant association between lifestyle factors and log-ferritin. Stepwise linear regression models for 5-year body weight gain were used for identifying significant lifestyle factors with predictive value.

Results A total of 1096 male workers with a baseline mean (SD) age of 32.5 (6.0) were included for analysis. Log-ferritin was positively associated with red meat intake, and negatively associated with blood donation, regular exercise at leisure time, and vegetarian diet. 5 -year body weight gain was associated with 12 hour shift work, and vegetarian diet after adjustment for age. In subgroup analysis among overweight males $(\mathrm{BMI}>=25)$ with non-alcoholic steatohepatitis (NASH), blood donation was significantly associated with less body weight gain in 5 years.

Conclusions 12-hour shift work, vegetarian diet may increase body weight in male workers. behaviours of health promotion, such as blood donation, regular exercise at leisure time, were associated with lower level of ferritin, a marker of total body iron load. Blood donation was the strongest factor associated with lower body weight gain within 5 years, especially among males with NASH. Physicians should recognise related behavioural factors for hyperferritinemia and body weight gain, and give high-risk individuals health counselling based on personalised risk profiles.

\section{INDICATORS TO SURVEILLANCE THE HEALTH OF NURSING STAFF}

'V E A F Felli, 'Baptista, ${ }^{2}$ Mininel, ${ }^{3}$ Sarquis. 'University of Sao Paulo - School of Nursing, Sao Paulo, Brazil; ${ }^{2}$ School of Nursing of Ribeirao Preto, University of Sao Paulo, Riberao Preto, Brazil; ${ }^{3}$ Nursing Department, Federal University of Parana, Curitiba, Brazil

10.1136/oemed-2013-101717.155
Objective Nursing morbidity profile has changed according to the working conditions. The profile that was composed of needlestick injuries and infectious diseases a few decades ago has nowadays become prevalent for musculoskeletal disorders. Knowing the health indicators of these workers is an essential tool to implement strategies to promote their health and monitor them. The aim of this study was to analyse health indicators related to nursing work, according to the institutional determinants and health problems.

Methods The study is characterised as epidemiological descriptive, cross-sectional and retrospective, and it was conducted in seven university hospitals in Brazil, in a universe of 44. From 2008 to 2009, information about the health problems of nursing staff-related work was sent by hospitals to researchers. The "System for Monitoring the Health of Nursing Workers (SIMOSTE)", that is an online system to capture data was used. For data analysis descriptive statistics was used. After the data analysis the System allows to verify the 13 proposed indicators. Results The indicators highlight regional disparities of the country. The indicators related to the work dynamics show, on average, that nursing staff represent $38.4 \%$ of the health team of the hospitals, nurses represent $21.8 \%$ of nursing team, and the ratio of 2.4 nursing staff per bed was found. Considering the indicators related to exposure to workloads and strain processes, the most often are the physiological workloads $(\mathrm{RR}=43.8)$, biological $(\mathrm{RR}=29.5)$ and psychic $(\mathrm{RR}=26.5)$; the health problems reported were musculoskeletal disorders (21.5\%), trauma, and external causes $(13 \%)$, mental and behavioural disorders $(8.3 \%)$. The indicator that relates the consequences show that in one year were registered more than 8,000 days lost at work.

Conclusion In synthesis, these indicators will allow the accompanying of effectiveness of the preventive measures adopted by the hospitals and the improvement of working conditions.

\section{WORKPLACE OCCUPATIONAL HEALTH INFORMATION}

KS Wilson, Darwin, Jones. NIOH, Johannesburg, South Africa

\subsection{6/oemed-2013-101717.156}

Objectives An information system on occupational hazards, injuries and near misses as well as disease and associated risk factors and prevention measures needed and/or implemented is essential for any large employer to effectively manage and mitigate injuries and diseases in work place. The information provided by a system is only as good as the information entered to the system thus staff attitude towards the system and surveillance is important to ascertain for the program to work effectively

Method A 15\% stratified random sample of employees was selected to participate in the baseline online survey. The questions covered incidents, workplace hazards and employee health. The employees were contacted via email with regular reminders to participate.

Results There were 314 participants giving a 32\% response rate. $23 \%$ of employees did not know or were unsure how to contact their safety health and environment officer. In the past 5 years $9 \%$ of staff have suffered needlesticks and the majority $(65 \%)$ reported the incident to both their supervisor and their health and safety representative. A few reported to a colleague or first aid provider and only 1 did not report. With other occupational injuries and diseases the patterns were the same. $60 \%$ of staff have received all three doses of the Hepatitis B vaccine and 26\% received flu vaccines. 
Conclusions The response rate was what was expected for this population and the majority indicated that they report all incidents which suggests that an online information system will be used and provide a valuable resource. With $40 \%$ of staff not complete with their Hepatitis B vaccines an information system will allow nurses to track these employees more effectively.

\section{Session: U. Cancer epidemiology III}

\section{OCCUPATION AND CANCER: AN ALTERNATIVE TOOL FOR EVALUATING THE EVIDENCES}

${ }^{1} \mathrm{O}$ E Oddone, ${ }^{2} \mathrm{C}$ P Crosignani, ${ }^{2} \mathrm{~S}$ A Scaburri, ${ }^{2} \mathrm{~B}$ E Bai, ${ }^{2} \mathrm{M}$ C Modonesi, 1 I M Imbriani. ${ }^{1}$ University of Pavia, Pavia, Italy; ${ }^{2}$ Italian National Cancer Institute, Milan, Italy

10.1136/oemed-2013-101717.157

Objectives Meta-analysis and pooled analysis are considered as a gold standard to summarise and to sift the biomedical evidences. In the framework of the Occupational Cancer Monitoring (OCCAM), it was developed a tool to assess the existence of an association between industrial sectors and cancer risk of a specific site so called "Literature Matrix" (LM), storing only positive literature results. The aim of this work is to challenge the adjusted results of this tool with those of meta-analysis studies.

Methods To evaluate the effectiveness of LM to provide useful summary risk estimates, we compared formal meta analyses with the set of "positive" results provided by the matrix. Among the several associations provided in LM, some were selected for present study. For this exploratory study we limited comparisons to 7 areas: agriculture and hematopoietic cancers as a whole, agriculture and non-Hodgkin lymphoma (NHL), agriculture and leukemia, agriculture and multiple myeloma, transports sector and female breast cancer (compared with two different meta-analysis), transports sector and lung cancer and painters and bladder cancer.

Results LM data, after adjustment with trim and fill method, show a substantial agreement with the correspondent meta-analysis, although results driven from LM data tend to an expected overestimation, but in most cases very slight. Results from LM data are always included between the limits of $95 \%$ CI of the correspondent meta-analysis, with the exception of Agriculture/NHL and Agriculture/All hematopoietic cancers. Results from LM data not adjusted are constantly farer from meta-analytic results, with the only exception of Painter/Bladder cancer analysis.

Conclusions The collection of only positive results derived from the scientific literature, and the use of an appropriate statistical correction, allows for a useful estimation of cancer risk by site and economic branch and it can be used for interpreting results of surveillance systems and for public health purposes.

\section{OCCUPATIONAL EXPOSURE TO CARCINOGENS IN DIFFERENT SUBTYPES OF SINONASAL CANCER. RESULTS FROM 100 CONSECUTIVE HOSPITAL BASED CASES IN ITALY}

${ }^{1} \mathrm{M}$ Bonzini, ${ }^{2}$ Parassoni, ${ }^{1}$ Facchinetti, ${ }^{1}$ Borchini, ${ }^{2}$ Turri Zanoni, ${ }^{2}$ Castelnuovo, 'Marco Mario. ${ }^{1}$ Epidemiology and Preventive medicine research Centre, University of Insubria, Varese, Italy; ${ }^{2}$ Othorinolaringology Unit, University of Insubria, Varese, Italy

\subsection{6/oemed-2013-101717.158}

Objective Sino-nasal cancer (SNC) is a rare and potentially fatal disease with a high occupational attributable fraction, being wood, leather dust, metals and solvents well recognised carcinogenic agents. The importance of occupational exposure varies across different histological subtypes and is disputed for tumors different from adenocarcinoma (AC). We quantified the etiological role of occupational exposure in a large hospital based cohort.

Methods We systematically evaluate 100 consecutive SNC cases after surgical treatment in Varese Hospital (Feb-2010/Oct-2012) through a standardised and validated questionnaire developed with the National Registry for SNC cases.

Results We evaluated 62 AC, 22 squamous-cell-carcinoma, 16 other (29\% women). Mean age at diagnosis was 63 . Aprevious occupational exposure was recognised for $59 \%$ of cases. The proportion of occupational cases was sensibly higher for AC $(83 \%)$ than for all other histotypes (19\%). For AC the recognized risk factors in the great majority of cases were leather and wood dusts (46\% both).

Conversely, for others histotypes different and less common exposures, such as chromium and formaldehyde, resulted more important. In particular we recognised 3 professional cases previously in the photolithographic industry. Mean latency period, 50 years $(\min 20-\max 72)$, and mean duration of exposure $=26 \mathrm{y}$ (min 4-max 54) were similar across histotypes.

Conclusions Occupational exposure plays a key role in the etiopathogenesis of SNC, not only for AC (with wood and leather dust that confirmed their impact in term of public health) but also for other histotypes with formaldehyde and chromium as important and often misrecognised occupational factors.

\section{NEW EPIDEMIOLOGICAL EVIDENCE ON THE HUMAN CARCINOGENIC POTENTIAL OF ACRYLONITRILE EXPOSURE}

${ }^{1} \mathrm{M}$ Marsh, ${ }^{1}$ Downing, 'Liu, ${ }^{2}$ Symons. ${ }^{1}$ University of Pittsburgh, Pittsburgh, United States of America; ${ }^{2}$ E. I. du Pont de Nemours and Company, Newark, United States of America

\subsection{6/oemed-2013-101717.159}

Objectives Acrylonitrile (AN) is an industrial chemical used in the production of fibres, plastics, synthetic rubbers, resins and pharmaceuticals. AN has been studied extensively in exposed workers and experimental animals, yet many questions remain regarding its carcinogenic potential in humans. In 1999, the International Agency for Research on Cancer (IARC) reclassified AN as a "possible" (Group 2B) human carcinogen based largely on findings from four epidemiological studies. AN is currently under review by the US Environmental Protection Agency and is a candidate for an upcoming IARC re-evaluation.

Methods The University of Pittsburgh, Department of Biostatistics, Center for Occupational Biostatistics and Epidemiology (COBE) is engaged in a series of epidemiology studies designed to advance knowledge regarding the human carcinogenicity of AN exposure. These studies are funded by the AN Group and INEOS Nitriles, Inc. and include:

An update and expansion of an earlier historical cohort study of workers exposed to AN at the Lima, $\mathrm{OH}$ production site owned by INEOS Nitriles. This site was one of eight AN production sites studied in the 1990s by the US National Cancer Institute (NCI) and independently studied by COBE in the same time period.

A sensitivity analysis based on the INEOS Lima cohort, designed to adjust lung cancer risks in relation to $\mathrm{AN}$ exposure for potential confounding by smoking. While individual-level smoking data are available for most subjects, these data appear to be severely misclassified, rendering them uninformative. 